\title{
Influence of weight ratio in polymer blend film on the phase separation structure and its optical properties
}

\author{
H.-M. Li, Z. Wang, X.-C. Chen, Z.-D. Xie, D.-J. Shu, M. Wang ${ }^{a}$, R.-W. Peng, and N.-B. Ming \\ National Laboratory of Solid State Microstructures and Department of Physics, Nanjing University, Nanjing 210093, P.R. China
}

Received: 6 September 2008 / Received in final form: 5 November 2008 / Accepted: 7 November 2008 Published online: 31 January 2009 - (c) EDP Sciences

\begin{abstract}
In this paper we report the nano-phase separation structure in the polymer blend film and its optical properties. Polystyrene (PS)/polymethyl methacrylate (PMMA) blend film is spin-coated on substrate. A sandwiched structure consisting of a depleted PMMA layer, a PS/PMMA blend layer and a PS-rich top layer has been formed. By selectively dissolving the PS-rich phase, a nanoporous film is generated, and the nanoporous structure can be tuned by changing the weight ratio of PS/PMMA in the blend solution in fabrication. The optical properties of the nanoporous thin films are determined. Our results show that by introducing the nanoporous structure, the refractive index can be effectively modified. By selecting proper film thickness, the maximum optical transmission can be achieved in the specific waveband.
\end{abstract}

PACS. 78.20.-e Optical properties of bulk materials and thin films - 61.43.Gt Powders, porous materials - 64.75.St Phase separation and segregation in thin films

\section{Introduction}

Phase separation of immiscible polymer blend has been intensively studied, especially in the system of PS/PMMA blend [1-18]. The phase separation process of polymer blend can be affected by a number of factors, such as solvent [1-3], substrate $[2,4,5]$, film thickness [6-9], molecular weight $[10,11]$, and blend ratio, etc. Essentially the phase separation process is determined by interfacial tension, solubility, combinational entropy of the polymers, etc. Depending on the control parameters, the variety of interfacial morphology of the phase separation is rich, changing from connected-islands, separated dots, pits, to lines. The spatial distribution of the phase separation patterns can be ordered $[1,5]$ or disordered, and the characteristic size of the structure can vary from a few nanometers to microns $[4,10-12]$. It has also been observed that when the molecular weight of the polymers is lower than a certain threshold, nanoporous structure is generated $[10,12,19,20]$.

On the other hand, it has been a desire for a long time in the community of optical materials to tune the effective refractive index of a solid thin film easily, for the purpose of generating antireflection films. Instead of changing the chemical components, recently it has been shown that introducing micro/nano structures may modify the refractive index of a material. Nowadays there are at least two major approaches to accomplish this strategy. One is to fabricate a "motheye" structure [21-25], and the other is to introduce nanoporous thin film on solid surface $[12,26-29]$. An antireflection film requires suitable

\footnotetext{
a e-mail: muwang@nju.edu.cn
}

film thickness and refractive index for the best performance. For example, the refractive index of the film, $n_{p}$, should satisfy $n_{p}=\left(n_{o} n_{s}\right)^{1 / 2}$, where $n_{p}, n_{o}, n_{s}$ represent the refractive index of the film, the environment and the substrate, respectively. For air-glass interface, the refractive index of the film should be 1.25 . However, such a low-refractive-indexed material is usually not easy to find in nature. The nanoporous structure provides an easy way to achieve such a material with reduced refractive index. Zhao et al. once investigated the influence of the fabrication technique on the porous size of the polymer nanoporous antireflection coating by atomic force microscopy (AFM) [30], yet the influence of weight ratio on the nano-phase separation structure was not included in previous studies.

In this paper, following the method reported by Walheim, etc. [12], we study the structure of nanophase separation with different weight ratio of PS/PMMA blends and the corresponding change of optical properties. The nanoporous thin film of PMMA is generated by selectively dissolving PS-rich phase from the spin-coated PS/PMMA blend film. It is shown that the porous structure in the film can be tuned by changing the weight ratio of PS and PMMA in preparing the blend solution. Consequently the optical properties of thin films can be effectively modified.

\section{Experiments}

Conventional spin-coating method is used to fabricate the thin film. Polymers with different molecular weight, $\mathrm{PS}\left(\mathrm{Mw}_{\mathrm{PS}}=10300\right)$ and PMMA $\left(\mathrm{Mw}_{\mathrm{PMMA}}=10600\right)$ 

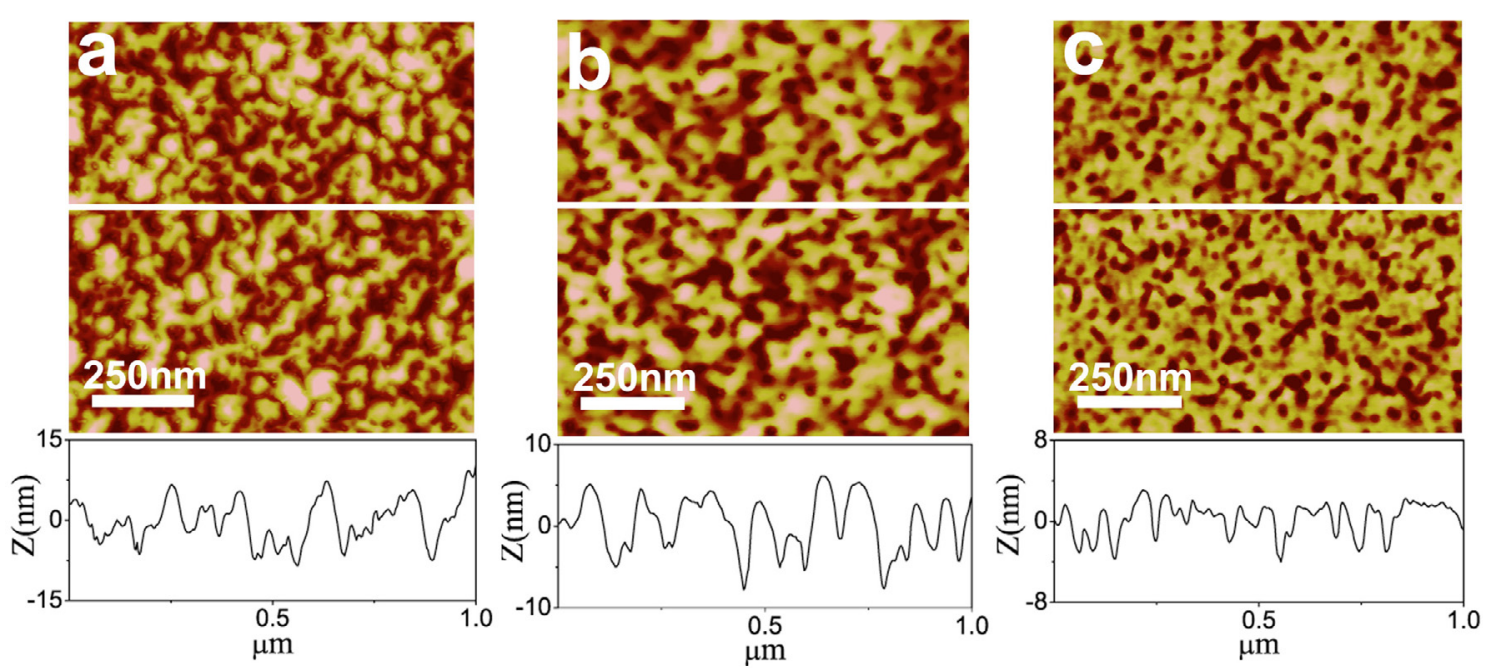

Fig. 1. (Color online) The surface morphology of PMMA nanoporous films after selective dissolving the PS-rich phase in the spin-coated PS/PMMA blend film. Figures 1a-1c correspond to the surface morphology generated at different PMMA-WF, $30 \%, 50 \%$ and $70 \%$, respectively. The depth profile along the marked line is provided below each micrograph.

(Mw stands for the weight-average molecular weight), are purchased from Fluka and used without further purification. The polydispersity indexes of PS and PMMA are 1.03, 1.06 respectively. The polymers were dissolved in analytic grade of tetrahydrofuran (THF). The concentration of polymers is $2.0 \mathrm{wt} \%$, yet the weight ratio of PS/PMMA varies. In our experiments, typically three types of blend solutions with PMMA weight fraction (PMMA-WF) of $70 \%, 50 \%$ and $30 \%$ are prepared. For the subsequent measurements of film structure and optical refractive index, commercially polished silicon wafers are used as the substrate. For transmission spectrum measurements, K9 optical glass plates $(n=1.567)$ are used as substrates in order to avoid unexpected adsorption of impurities. Conventional cleaning processes for silicon wafers and glass plates are applied. The cleaned substrates are rinsed thoroughly with deionized water (Millipore, $18.2 \mathrm{M} \Omega \mathrm{cm}^{-1}$ ) and dried in $\mathrm{N}_{2}$. The blend thin film is spin-coated in nitrogen atmosphere at $3000 \mathrm{rpm}$ for $2.5 \mathrm{~min}$. PS-rich phase in the thin film is thereafter removed with cyclohexane and dried with $\mathrm{N}_{2}$. In this way a thin film with nanoporous structure is achieved. In order to get more evident effect in transmission measurements, glass substrate has been spin-coated on both sides.

The surface morphology of the nanostructured film is measured by atomic force microscopy (AFM) in tapping mode (Nanoscope IIIa). The cross-section of the nanoporous film is observed with a field emission scanning electron microscope (FESEM, LEO-1530VP). For this purpose, the sample is immersed in liquid nitrogen first and cracked immediately after removing it out of liquid nitrogen. In this way we suppose that the nanoporous structure in the polymer film is preserved without much elastic deformation.

The thickness and optical-dispersion relation of the nanoporous films are measured by an ellipsometer (GES5 SOPRA). The incident angle is $75^{\circ}$ and the wavelength range is $300-800 \mathrm{~nm}$ (with a step of $10 \mathrm{~nm}$ ). The fit- ting model is air/single layer film /Si (100) and the optical constants are described by Cauchy dispersion model. In our experiment, the standard deviations are all below $2.5 \times 10^{-3}$. Transmission spectra of the nanoporous films are measured by a UV/VIS/NIR Spectrometer (Lambda 900, Perkin Elmer) in the waveband of 300-850 nm.

\section{Results and discussion}

AFM micrographs of the sample surface with PMMA-WF $30 \%, 50 \%$, and $70 \%$ are shown in Figure 1, where the PS-rich phase has been chemically removed. Nanoporous structures can be clearly identified. By increasing PMMA-WF, the morphology of the PMMA-rich phase transits from the separated islands surrounded by air (Fig. 1a) to a continuous PMMA film decorated with tiny air holes (Fig. 1c). Such a tendency can also be seen in the line profiles of the AFM pictures. One may estimate the depth of the nanoholes from the line profile, which is of the order of ten nanometers. By increasing PMMA-WF, the depth of the holes decreases in average.

The cross-section of the nanoporous film observed by FESEM is shown in Figure 2, where PMMA-WF is $30 \%$. One may find that the nanopores penetrate through the film, and the depth of the holes may reach nearly $80 \mathrm{~nm}$, which is deeper than that measured by AFM (Fig. 1). A possible explanation could be that in AFM measurement, due to the restriction of the tip-size of AFM probe, the depth of the holes becomes shallower than that observed by SEM.

The formation process of the nanoporous film can be understood as follows. Since THF is a better solvent for PS than for PMMA, a PMMA-rich phase is formed earlier due to precipitation in the spin-coating process. In addition, PMMA is more hydrophilic to silicon substrate. Therefore during the spin-coating, a thin layer of PMMA is easily formed on silicon substrate. At the same time, 


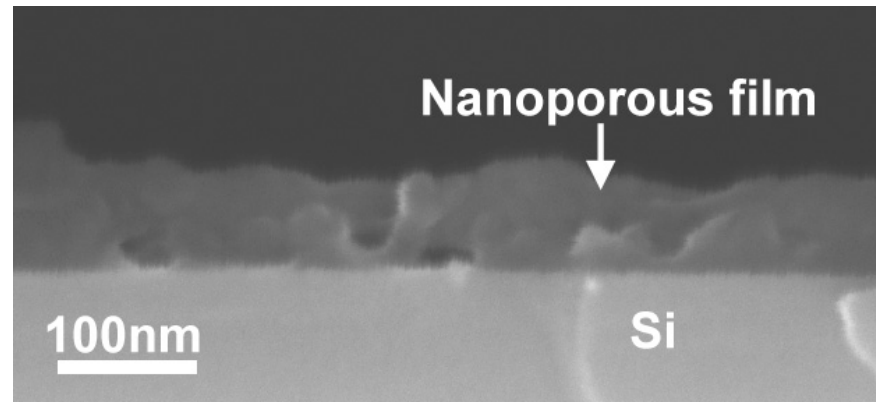

Fig. 2. FESEM micrograph of the cross-section of the nanoporous film generated on a silicon substrate. The PMMA$\mathrm{WF}$ in preparation of this film is $30 \%$.

Table 1. The parameters of nanoporous films with different PMMA-WF in preparation.

\begin{tabular}{ccccc}
\hline PMMA-WF (\%) & $n_{p}$ & $t_{0}(\mathrm{~nm})$ & $t(\mathrm{~nm})$ & $t_{\mathrm{PS}}(\mathrm{nm})$ \\
\hline 70 & 1.412 & $100 \pm 1$ & $68 \pm 1$ & $32 \pm 2$ \\
50 & 1.323 & $118 \pm 1$ & $105 \pm 1$ & $13 \pm 2$ \\
30 & 1.232 & $104 \pm 1$ & $100.4 \pm 1$ & $3.6 \pm 2$ \\
\hline
\end{tabular}

$t_{0}$ is the thickness of the blend film as spin-coated, $t$ is the thickness of the nanoporous film, $t_{P S}$ is the calculated PS-rich top layer thickness by the equation: $t_{P S}=t_{0}-t$.

the PS-rich phase tends to aggregate to the film surface due to its lower surface energy. Yet due to the segregation effect, small amount of PMMA remains in PS-rich phase and small amount of PS remains in PMMA-rich phase. For these reasons, a sandwiched structure (depleted PMMA layer/PS-PMMA blend layer/PS-rich top layer) is formed. In order to prove the existence of the PS-rich top layer, the thicknesses of both the blend film as spin-coated and the corresponding nanoporous film are measured (Tab. 1). When the PMMA-WF is $30 \%$, the PS content is excessive, a thicker PS-rich top layer will be formed, and in the PS/PMMA blend layer, the dispersed PMMA-rich phase is formed. By increasing PMMA-WF, the PS-rich top layer becomes thinner and thinner. When the PMMA-WF is $70 \%$, the PS-rich top layer vanishes and the PS-rich phase disperses in the PMMA matrix. This explains that by removing the PS-rich phase, the PMMA-rich phase transits from the dispersed phase (Fig. 1a) to the continuous phase (Fig. 1c).

The optical transmission spectra of the glass plates coated with nanoporous films on both sides are shown in Figure 3. For comparison, the transmission of a bare glass plate is also measured, which was about $92 \%$ (Fig. 3, diamond). By decreasing PMMA-WF, we find that the transmission of the nanoporous film increases. For PMMA-WF $30 \%$, the transmission reaches $99.5 \%$ (Fig. 3, triangle). In order to understand the optical properties of the nanoporous film, the optical-dispersion relation is determined by ellipsometer. The measured optical-dispersion relation of nanoporous films are shown in Figure 4. One may find that the refractive index does not change significantly as a function of wavelength. For this reason, we take the refractive index at the wavelength $632 \mathrm{~nm}$ as the

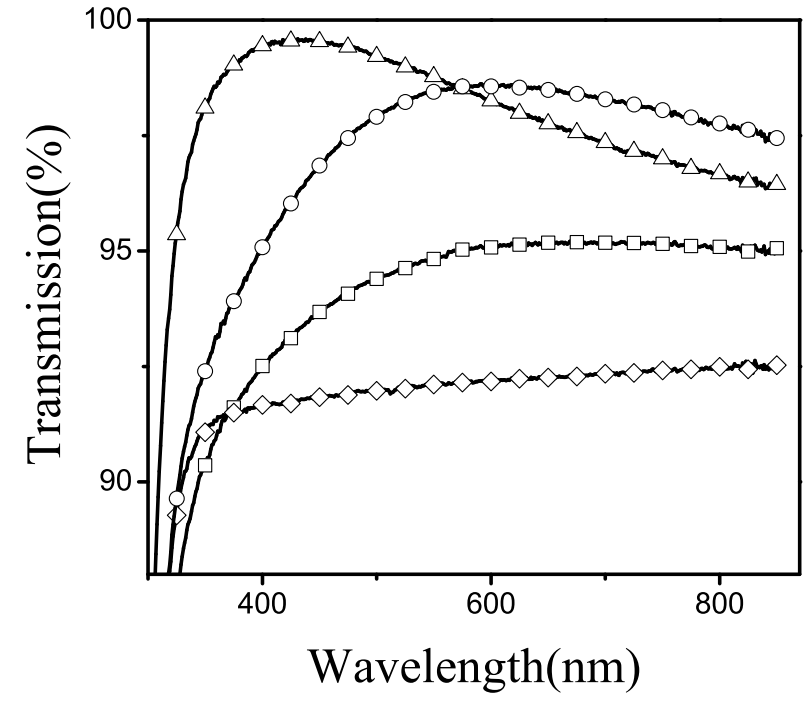

Fig. 3. The measured transmission spectra of the nanoporous films with PMMA-WF 30\% (triangle), 50\% (circle), and 70\% (square) in the blend for spin-coating. The transmission of a bare K9 optical glass plate was measured (diamond), which is used as a reference.

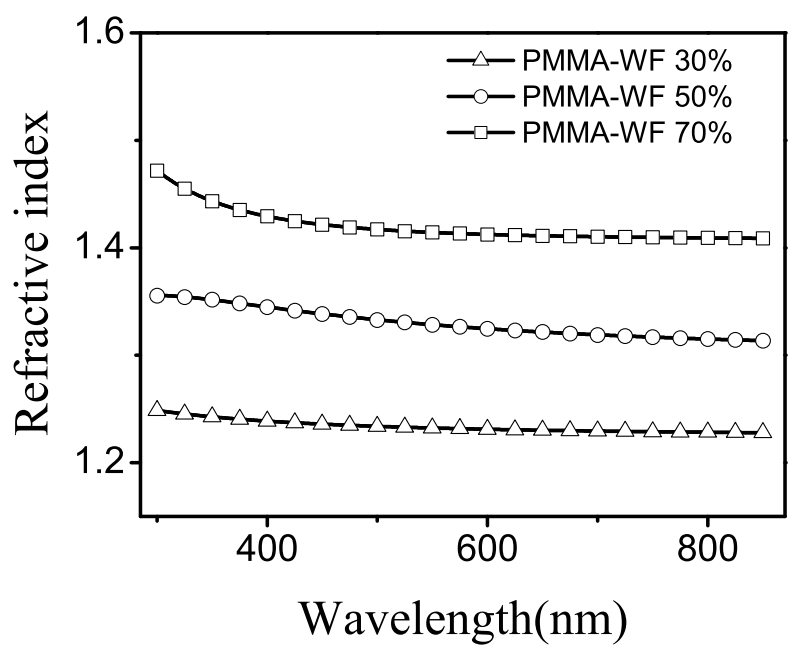

Fig. 4. The optical-dispersion relation of the nanoporous films measured by ellipsometer when PMMA-WF was 70\%, 50\%, and $30 \%$ in the blend for spin-coating, respectively. By decreasing PMMA-WF, the refractive index of the nanoporous film can be effectively decreased.

representative value of the coating film. The results are listed in Table 1. By decreasing PMMA-WF, the refractive index of the nanoporous film decreases. Because the size of the nanopores is much smaller than the wavelength, the nanoporous film can be considered as a continuous, homogeneous media. In fact, the refractive index of a material depends on its density. By introducing nanoporous structure, the average material density is decreased, so does the refractive index [31]. Furthermore, by decreasing PMMA-WF, the porosity of the film (defined as the volume fraction of the air in the porous film) increases, which effectively decreases the refractive index. 
As an antireflection film, two essential requirements should be satisfied. One is that the refractive index of the film should satisfy $n_{p}=\left(n_{o} n_{s}\right)^{1 / 2}$. For a film between air and K9 glass substrate, the refractive index should be 1.25. The second condition is that the optical thickness of the film should be a quarter of the incidence wavelength $(\lambda)$, i.e., $2 n_{p} t=\lambda / 2$, so the reflected light will be in antiphase with that of the incidence light ( $t$ represents the film thickness). Due to the destructive interference of reflection light on two interfaces of the coating layer, the reflection is suppressed. For the scenario of PMMA-WF $30 \%$, the refractive index is 1.232 (Tab. 1), which is very close to the ideal value for a perfect antireflection film. As a result, transmission can reach as high as $99.5 \%$ (Fig. 3, triangle). When PMMA-WF increases, the refractive index of the nanoporous film also increases. Meanwhile the first requirement for the maximum transmission cannot be satisfied. Hence the transmission decreases (Fig. 3, square and circle). One may note that in the transmission spectrum there exists a bump, and the position of the bump shifts as PMMA-WF is changed. We suggest that this effect is associated with the thickness of the anti-reflection coating. The optical thickness of the nanoporous film is determined by the product of refractive index and the layer thickness of the nanoporous film. When PMMA-WF is $30 \%$, its refractive index is low and the film is thin. Therefore the wavelength satisfying anti-reflection condition, $2 n_{p} t=\lambda / 2$, is short. When PMMA-WF increases, both the thickness and the refractive index of the coating film are increased. Hence the wavelength satisfying the best anti-reflection condition shifts to a higher value, as that shown in Figure 3. In order to control the position of the bump to the required wavelength, one may control the film thickness by repeated spin-coating. Figure 3 demonstrates clearly that only when the requirements for both refractive index and film thickness are satisfied, the maximum transmission can be achieved.

\section{Conclusion}

We demonstrate in this paper the effect of weight ratio of PS/PMMA blend on the phase separation structures in a spin-coated thin film. By increasing PMMA-WF, the thickness of the PS-rich top layer decreases and the PMMA-rich phase evolves from a dispersed phase to a continuous phase in the blend layer. After removing the PS-rich phase, a porous film with lower effective refractive index is generated on the solid surface. The maximum transmission can be achieved at the desired wavelength by selecting a proper film thickness. Our studies demonstrate that this is an easy and effective way to fabricate anti-reflective coating.

This work has been supported by the grants from the Ministry of Science and Technology of China (Nos. 2004CB619005, 2006CB921804), the National Science Foundation of China (Nos. 10874068, 10625417) and Jiangsu Province (No. BK2008012). Authors also thank Prof. Cai-Hua Zhou for help in sample preparation.

\section{References}

1. E. Kumacheva, L. Li, M.A. Winnik, D.M. Shinozaki, P.C. Cheng, Langmuir 13, 2483 (1997)

2. S. Walheim, M. Böltau, J. Mlynek, G. Krausch, U. Steiner, Macromolecules 30, 4995 (1997)

3. L. Cui, Y. Ding, X. Li, Z. Wang, Y.C. Han, Thin Solid Films 515, 2038 (2006)

4. I. Prosycevas, S. Tamulevicius, A. Guobiene, Thin Solid Films 453, 304 (2004)

5. M. Böltau, S. Walheim, J. Mlynek, G. Krausch, U. Steiner, Nature 391, 877 (1998)

6. X. Li, Y.C. Han, L.J. An, Appl. Surf. Sci. 230, 115 (2004)

7. C. Ton-That, A.G. Shard, D.O.H. Teare, R.H. Bradley, Polymer 42, 1121 (2001)

8. K. Tanaka, A. Takahara, T. Kajiyama, Macromolecules 29, 3232 (1996)

9. K. Tanaka, J.S. Yoon, A. Takahara, T. Kajiyama, Macromolecules 28, 934 (1995)

10. X. Li, Y.C. Han, L.J. An, Polymer 44, 8155 (2003)

11. X. Li, R.B. Xing, Y. Zhang, Y.C. Han, L.J. An, Polymer 45, 1637 (2004)

12. S. Walheim, E. Schäffer, J. Mlynek, U. Steiner, Science 283, 520 (1999)

13. C. Ton-That, A.G. Shard, R. Daley, R.H. Bradley, Macromolecules 33, 8453 (2000)

14. M. Harris, G. Appel, H. Ade, Macromolecules 36, 3307 (2003)

15. S.Y. Heriot, R.A.L. Jones, Nat. Mater. 4, 782 (2005)

16. K. Dalnoki-Veress, J.A. Forrest, J.R. Dutcher, Phys. Rev. E 57, 5811 (1998)

17. J. Kressler, N. Higashida, K. Shimomai, T. Inoue, T. Ougizawa, Macromolecules 27, 2448 (1994)

18. J. Raczkowska, J. Rysz, A. Budkowski, J. Lekki, M. Lekka, A. Bernasik, K. Kowalski, P. Czuba, Macromolecules 36, 2419 (2003)

19. M.S. Park, J.K. Kim, Langmuir 21, 11404 (2005)

20. K. Biswas, S. Gangopadhyay, H.C. Kim, R.D. Miller, Thin Solid Films 514, 350 (2006)

21. Y. Kanamori, K. Hane, H. Sai, H. Yugami, Appl. Phys. Lett. 78, 142 (2001)

22. Y. Kanamori, M. Sasaki, K. Hane, Opt. Lett. 24, 1422 (1999)

23. M.W. Cao, X.Y. Song, J. Zhai, J.B. Wang, Y.L. Wang, J. Phys. Chem. B 110, 13072 (2006)

24. C. Aydin, A. Zaslavsky, G.J. Sonek, J. Goldstein, Appl. Phys. Lett. 80, 2242 (2002)

25. M.J. Minot, J. Opt. Soc. Am. 66, 515 (1976)

26. Z.Z. Wu, J. Walish, A. Nolte, L. Zhai, R.E. Cohen, M.F. Rubner, Adv. Mater. 18, 2699 (2006)

27. J. Hiller, J.D. Mendelsohn, M.F. Rubner, Nat. Mater. 1, $59(2002)$

28. H.Y. Koo, D.K. Yi, S.J. Yoo, D.-Y. Kim, Adv. Mater. 16, 274 (2004)

29. H.C. Kim, J.B. Wilds, C.R. Kreller, W. Volksen, P.J. Brock, V.Y. Lee, T. Magbitang, J.L. Hedrick, C.J. Hawker, R.D. Miller, Adv. Mater. 14, 1637 (2002)

30. M. Zhao, Z.Y. Yang, D.Q. Zhu, X. Jin, D.X. Huang, J. Opt. Soc. Am. B 22, 1330 (2005)

31. M.D. Morariu, Ph.D. thesis, University of Groningen, The Netherlands, 2004 